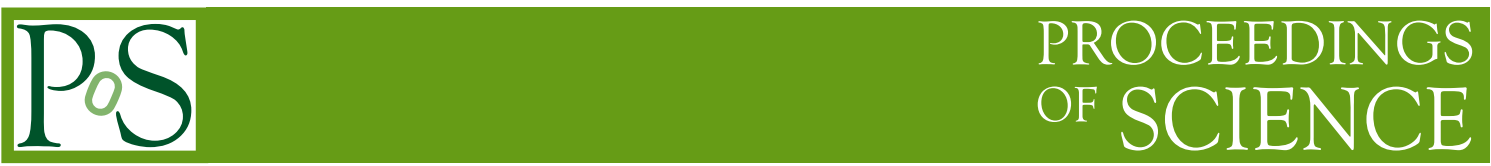

\title{
Recent results of the ANTARES neutrino telescope
}

\author{
Tommaso Chiarusi $^{a, *}$ and the ANTARES Collaboration \\ a INFN-Sezione di Bologna, \\ Viale Berti-Pichat 6/2, 40127 Bologna, Italy \\ E-mail: tommaso.chiarusi@bo.infn.it
}

The ANTARES detector is the first Cherenkov neutrino telescope realised in the Mediterranean sea. It is continuously taking data since 2007, with the primary aim to detect astrophysical neutrinos in the TeV-PeV range. A very good angular resolution in all flavour neutrino interaction channels, together with the depth of the abyssal site ( $2500 \mathrm{~m}$ below the sea level) led to an unprecedented sensitivity in the searches for neutrino sources in the Southern Sky and in the energy range below $100 \mathrm{TeV}$. This has allowed constraining the origin of the cosmic neutrino flux discovered by the IceCube detector. ANTARES has also implemented a rich multi-messenger program, providing with both online and offline pipelines for alert sending and follow-up observations of interesting astrophysical events. Among these, the searches triggered by gravitational wave observations are of prominent interest. Other physics topics are also covered: searches of dark matter annihilation or decay in massive objects; the search for relic massive magnetic monopoles and nuclearites; the study of atmospheric neutrinos and neutrino oscillations.

40th International Conference on High Energy physics - ICHEP2020

July 28 - August 6, 2020

Prague, Czech Republic (virtual meeting)

\footnotetext{
${ }^{*}$ Speaker
} 


\section{Introduction}

Multi-TeV neutrinos are expected to be emitted in a large sample of astrophysical objects: together with gamma-rays, they are produced in the interactions of accelerated nuclei with matter and radiation at the sources or along their acceleration path. Since their first detection by IceCube [1] in 2013, neutrino Astronomy is fully part of the multimessenger approach, which includes gravitational waves and also other form of radiations. ANTARES and KM3NeT (also reported in these preoceedings [2]) neutrino telescopes play a major role for complementing the IceCube searches at South Pole. As installations in the Mediterranean Sea, they benefit of optimal optical properties of seawater which allow unprecedented pointing and reconstruction performance. In this contribution, we report the status of the ANTARES detector, still operating since more than 12 years, and its main scientific achievements.

\section{The ANTARES detector and its performances}

The ANTARES (Astronomy with a Neutrino Telescope and Abyss environmental Research) detector [3] is the largest neutrino telescope operating undersea. It is installed at a depth of $2475 \mathrm{~m}$ in the Mediterranean Sea, about $40 \mathrm{~km}$ off the French coast near Toulon (France), and is running since 2007. It consists of an array of 885 optical modules, each one equipped with one 10-inch Photomultiplier (PMT) oriented at $45^{\circ}$ downward, and distributed in triplets along 12 mooring lines mutually distant of about $70 \mathrm{~m}$. The corresponding instrumented volume is about $0,05 \mathrm{~km}^{3}$. The power distribution and data transfer are granted by an electro-optical cable deployed from the shore-station to the underwater detector. ANTARES records the Cherenkov light induced along the path in water by the charged particles produced in the interaction of a high energy (HE) neutrino in the vicinity of the detector. Two classes of events are set: muon track-like events from $v_{\mu}$ chargedcurrent interactions; cascade-like events from all other (charged- and neutral-current) interaction channels. The time, position and amplitude of the recorded signals on the PMTs are used to estimate the energy and the direction of the incident neutrino. The optical properties of sea water allow a very good angular resolution for the reconstructed direction of detected neutrino candidates for all event topologies: $\sim 0.4^{\circ}$ for tracks above $10 \mathrm{TeV}$, and $\sim 3^{\circ}$ for showers. Such a reconstruction performance also relies on the detector accuracy in the measurement of the hits arrival time $(\sim 1$ ns), provided by insitu array of laser and LED calibration beacons, and position $(\sim 10 \mathrm{~cm})$, via the dedicated acustic positioning system. Using data collected in the period of 2007-2016, the absolute pointing performance of the detector has been validated by measuring the deficit of atmospheric muons in correspondance with the direction of the Moon, the so called Moon shadow which was observed with a statistical significance of $3.5 \sigma$ [4].

\section{Diffuse flux searches}

A neutrino diffuse flux can originate from the ensemble of unresolved individual sources, too faint to be detected, by cosmic ray propagation effects or by a combination of these processes. The IceCube findings mentioned in the opening of these proceedings concern to a highly-significant excess compatible with the hypothesis of an all-flavour neutrino flux isotropically distributed over 
the whole sky. Although the measured energy spectra fits to an unbroken single-power-law of the kind $d \Phi(E) / d E=\Phi_{0} E^{-\Gamma}$, some tension is present between the results obtained independently with the two principal data samples: the High Energy Starting Events (mainly cascades pointing to the Southern sky) producing a softer spectrun $(\Gamma \sim 2.5)$ and the Track-like Events ( upwardgoing muons from the Nother sky) giving a harder spectrum $(\Gamma \sim 2.1)$.

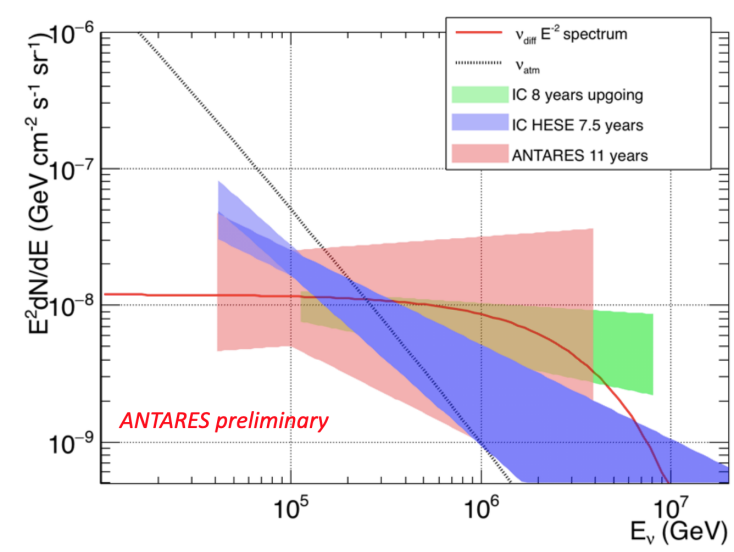

Figure 1: Comparison of the power-law one-flavour neutrino flux as determined by ANTARES, light red band, and IceCube, green band for upgoing tracks, blue band for HESE events. The conventional+prompt atmospheric neutrino flux and expected $E^{-2}$ cosmic diffuse flux with $3 \mathrm{PeV}$ cutoff are superimposed for reference.

ANTARES events, collected during 11 years of data taking from 2007 and 2018 [5], offer the opportunity to perform a complementary search. After energy-related selection cuts, a total of 50 ( 27 track-like +23 shower-like) events are observed in data, with $36.1 \pm 8.7$ being expected from pure background. A maximum-likelihood method is applied to fit the observed distributions of the energy estimators. The best-fit cosmic flux yields a single-flavor normalization at $100 \mathrm{TeV}$ equal to $\Phi_{0}^{1 f}(100 \mathrm{TeV})=1.5 \pm 1.0 \times 10^{-18} \mathrm{GeV}^{-1} \mathrm{~cm}^{-2} \mathrm{~s}^{-1} \mathrm{sr}^{-1}$ and a spectral index $\Gamma=2.3_{-0.4}^{+0.4}$. Although the null cosmic flux hypothesis can't be excluded with a large significance $(1.8 \sigma)$, the ANTARES result is inline with the hypothesis that the IceCube flux has a cosmic origin. Figure 1 shows the one-flavour neutrino flux obtained from ANTARES measurements compared to IceCube fluxes for upgoing tracks and HESE events as reported in [6, 7]. An ANTARES and IceCube joint analysis was performed to constrain the "KRA $\gamma$ " model [8], which accounts for local cosmic ray flux measurements as well as Galactic $\gamma$-ray observations. By combining the 10 years track and cascades events recorded by ANTARES and the 7 years track sample of IceCube, no significant excess was found to justify the mentioned "spectral anomaly" between the Southern and Northern emisphere.

\section{Point sources searches}

A recent search for point-like and extended sources of cosmic neutrinos in the Southern Sky was performed by combining data collected by the ANTARES and IceCube neutrino telescopes [9]. The ANTARES data set consists of nine years of track-like and shower-like events pointing 
in the direction of the Southern hemisphere, selected for point-source detection. IceCube data set concerns the throughgoing track-like events used in the seven-year IceCube point-source search. Compared to both individual analyses, the combination of the two detectors, which differ in size and location, allows for an improvement of up to a factor $\sim 2$ in the sensitivity in different regions of the Southern Sky, depending on the energy spectrum of the source. Figure 2 reports, as function of the source declination, and for hard (left) and soft (right) assumption on the unbroken $E_{v}^{\gamma}$ neutrino spectral index, the point-source sensitivity for both individual and combined analyises, together with the combined $90 \%$ upper limits for a selection of sources. For a soft spectral index, the contribution of high-energy neutrinos is suppressed and ANTARES dominates in most of the Southern Sky.
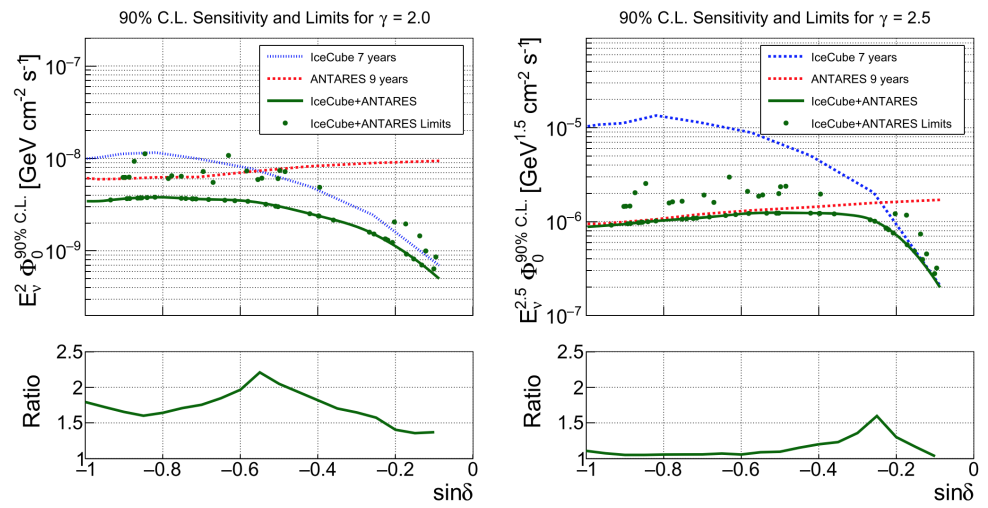

Figure 2: Top panels: combined 90\% C.L. upper limits (dots) and sensitivity (solid lines) to the one-flavor neutrino flux versus the source declination. Results shown for spetral index $\gamma=2.0$ (left) and $\gamma=2.5$ (right). The dashed curves indicate the sensitivities for the IceCube (blue) and ANTARES (red) individual analyses. Bottom panels: ratio between the best individual sensitivity and the combined sensitivity as a function of the source declination.

The 11-years ANTARES data set has been also used to look for a steady emission of neutrinos from the direction of the blazar TXS 0506+056 [10], being considered the first high-energy neutrino source ever found, as published by IceCube in [11]. No significant evidence of cosmic neutrinos is found, with a total number of fitted signal events $\mu_{\text {sig }}=1.03$ at the location of the blazar and a p-value of $3.4 \%$ for an unbroken power-law spectrum $E^{-2.0}$.

\section{Dark matter searches}

Neutrinos are expected to be one of the annihilation signatures of Weakly Interactive Massive Particles (WIMPs) trapped in massive astrophysical objects. The Sun and the Galactic Centre represent two particularly interesting dark matter source candidates for ANTARES since the latitude of the detector location offers an observation of the Sun with less atmospheric background and a privileged visibility of the Galactic Centre. Figure 3 shows the latest results of a joint analysis with ANTARES and IceCube datasets [12] for WIMP neutrinos from the Galactic Centre, compared to those of other experiments. Searchs for WIMP from the Sun and Earth centre where already reported in $[13,14]$. 


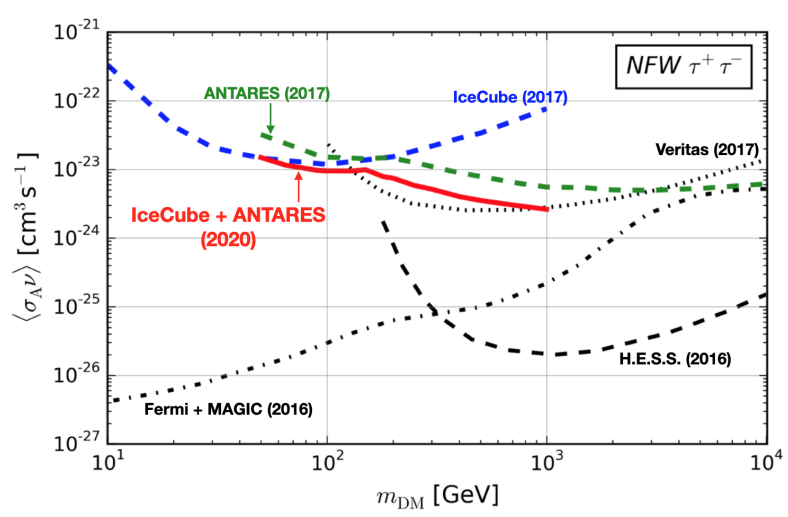

Figure 3: $90 \%$ C.L. upper limit on the thermally averaged dark matter annihilation cross section $\left\langle\sigma_{A} v\right\rangle$ obtained for the combined analysis as a function of the dark matter mass $m_{D M}$. The limits from IceCube and ANTARES individaully and from VERITAS, Fermi+MAGIC, and H.E.S.S. limits are also shown (references are reported in [12])

\section{The multi-messenger program}

An intense multi-messenger program is set by the ANTARES Collaboration on two fronts: distributing alerts to optical robotic telescopes (TAROT, ROTSE and MASTER), radio (MWA), X-ray (Swift, INTEGRAL) and $\gamma$-ray (H.E.S.S., HAWC) telescopes; following up external alerts from the Gamma-ray Coordinated Network to look for transient events. In addition to that, several on- and off-line studies have also been conducted to search for neutrinos in coincidence with the gravitational wave events recently detected by LIGO and Virgo [15] and [16-18]. All the above analyses didn't produced any evidence of neutrino candidates. It is worthy to mention three followup analyses that were performed between 2017 and 2018, initially triggered by the GCN circular [19], reporting about the extremely high energy cosmic neutrino IC170922A. None of the three analyses (online, time independent and time dependent point-source approaches) yielded neutrino events, as reported in [10]. Recently, an ANTARES study aimed at constraining a possible origin of the IceCube astrophysical signal from transient sources [20]. The ANTARES data-set was scanned to look for time and space correlation with more than 50 IceCube track-like events. Each IceCube event was treated as a potential transient neutrino source. Although neutrino arrival times were used to enhance the discovery potential, no ANTARES event was observed in correlation with the IceCube candidates, and upper limits on the neutrino fluence from the investigated candidates were set. Finally, ANTARES has also looked for neutrinos in coincidence with 12 Fast Radio Bursts [21] : also in this case no coincident neutrino candidate is observed, and constraints on the neutrino fluence and on the energy released are derived from the associated null results.

\section{Conclusions}

The ANTARES detector has been continuously taking data since more than 12 years. The obtained science results are competitive and contribute significantly to a wide range of Astrophysics and Physics searches. In addition to the topic reported in these Proceedings, it is worthy also to 
mention the recent atmospheric neutrino oscillation searches [22] and the magnetic monopole searches [23]. The complementarity of ANTARES with respect to IceCube demonstrates the huge potential of KM3NeT, the next generation Mediterranean Cherenkov telescope, expected to open a new era of neutrino Astronomy.

\section{References}

[1] M.G. Artsen et al., Science 342 (2013) .

[2] F. Huang, (KM3NeT Collaboration) in these Proceedings.

[3] M. Ageron et al., Nucl. Instrum. Meth. A 656 (2013) 11.

[4] A. Albert et al., Eur.Phys.J. 78 (2018) 1006.

[5] L. A. Fusco, F. Versari (ANTARES Collaboration) PoS(ICRC2019) (2019) 981.

[6] IceCube Collaboration PoS(ICRC2019) (2019) 1017.

[7] IceCube Collaboration PoS(ICRC2019) (2019) 1004.

[8] A. Albert et al., The Astrophysical Journal Letters 868 (2018) L20.

[9] A. Albert et al.The Astrophysical Journal 892 (2020) 92(12pp).

[10] A. Albert et al.The Astrophysical Journal Letters 863 (2018) L30.

[11] M.G. Artsen et al., Science 361 (2018) 147.

[12] A. Albert et al.Physical Review D 102 (2020) 082002.

[13] S. Adrián-Martínez et al.Physics Letters B 759 (2016) 69.

[14] A. Albert et al.Physics of the Dark Universe 16 (2017) 41.

[15] A. Albert et al.Eur.Phys.J. C 80 (2020) 487.

[16] A. Albert et al.The Astrophysical Journal Letters 850 (2017) L35.

[17] A. Albert et al.Phys. Rev. D 96 (2017) 022005.

[18] S. Adrián-Martínez et al.Phys. Rev. D 93 (2016) 122010.

[19] GCN circular 21916, 2017.

[20] A. Albert et al.The Astrophysical Journal 879 (2019) 108.

[21] A. Albert et al., Mon. Not. Roy. Astron. Soc. 842 (2019) 184.

[22] A. Albert et al.J. High Energ. Phys. 2019 (2019) 113.

[23] A. Albert et al.J. High Energ. Phys. 2017 (2017) 54. 